\title{
MEASURE INEQUALITIES AND THE TRANSFERENCE THEOREM IN THE GEOMETRY OF NUMBERS
}

\author{
CHENGLIANG TIAN, MINGJIE LIU, AND GUANGWU XU
}

(Communicated by Edward C. Waymire)

\begin{abstract}
The measure inequalities of Banaszczyk have been important tools in applying discrete Gaussian measure over lattices to lattice-based cryptography. This paper presents an improvement of Banaszczyk's inequalities and provides a concise and transparent proof. This paper also generalizes the transference theorem of Cai to general convex bodies. The bound is better than that obtained by simply generalizing the $l_{2}$ norm using the canonical norm inequalities.
\end{abstract}

\section{INTRODUCTION}

An $n$-dimensional lattice $L \subset \mathbb{R}^{n}$ is an additive subgroup of $\mathbb{R}^{n}$ generated by the set of $n$ linearly independent vectors $\mathbf{b}_{1}, \cdots, \mathbf{b}_{n}$, which is called a basis of $L$. The family of all $n$-dimensional lattices in $\mathbb{R}^{n}$ is denoted by $\mathcal{L}_{n}$. The dual of a lattice $L$ is the set

$$
L^{*}=\left\{\mathbf{x} \in \mathbb{R}^{n}:\langle\mathbf{x}, \mathbf{y}\rangle \in \mathbb{Z} \text { for each } \mathbf{y} \in L\right\},
$$

where $\langle\mathbf{x}, \mathbf{y}\rangle$ is the canonical inner product in $\mathbb{R}^{n}$. The basis $\mathbf{b}_{1}^{*}, \cdots, \mathbf{b}_{n}^{*}$ of $L^{*}$ satisfying $\left\langle\mathbf{b}_{i}, \mathbf{b}_{j}^{*}\right\rangle=\delta_{i j}$ is called the dual basis of $\mathbf{b}_{1}, \cdots, \mathbf{b}_{n}$ where $\delta_{i j}(1 \leq i, j \leq n)$ is the Kronecker symbol.

A convex body in $\mathbb{R}^{n}$ is a compact convex subset of $\mathbb{R}^{n}$ containing interior points. The family of all convex bodies in $\mathbb{R}^{n}$ which are symmetric with respect to the origin is denoted by $\mathfrak{C}_{n}$. Given a convex body $U \in \mathfrak{C}_{n}$, we define the polar body $U^{\circ}$ as follows:

$$
U^{\mathrm{o}}=\left\{\mathbf{x} \in \mathbb{R}^{n}:|\langle\mathbf{x}, \mathbf{y}\rangle| \leq 1 \text { for each } \mathbf{y} \in U\right\} .
$$

Let $\|\mathbf{x}\|_{U}=\inf \{r \geq 0: \mathbf{x} \in r U\}$ denote the norm on $\mathbb{R}^{n}$ induced by $U$, and let $d_{U}$ denote the metric induced by $\|\cdot\|_{U}$. The $i$ th successive minima of $L$ with respect to $U$ is defined as

$$
\lambda_{i}(L, U)=\min \{r>0: \operatorname{dim} \operatorname{span}(L \cap r U) \geq i\} .
$$

Received by the editors August 6, 2011 and, in revised form, February 2, 2012 and February 26, 2012.

2010 Mathematics Subject Classification. Primary 06D50, 11H06; Secondary 03G10, 52C07.

Key words and phrases. Convex body, lattice-based cryptography, Gaussian measures, transference theorem.

The first author was supported by the National Natural Science Foundation of China (Grant No. 61133013 and No. 60931160442).

The second author was supported by Tsinghua University Initiative Scientific Research Program No. 2009THZ01002. 
For each $U \in \mathfrak{C}_{n}$, consider the quantity

$$
\xi(U)=\sup _{L \in \mathcal{L}_{n}} \max _{1 \leq i \leq n} \lambda_{i}(L, U) \lambda_{n-i+1}\left(L^{*}, U^{0}\right) .
$$

Determining the upper bound of $\xi(U)$ is known as the so-called transference theorem and is a classical problem in the geometry of numbers. It has been a topic of intensive investigation. For more details, see [3] and the references therein. Let us mention only the best known bounds here. In [3], Banaszczyk proved that for any positive integer $n$ and the Euclidean closed unit ball $\mathcal{B}_{2}^{n}, \xi\left(\mathcal{B}_{2}^{n}\right) \leq n$. Furthermore, for $n$ large enough,

$$
\xi\left(\mathcal{B}_{2}^{n}\right) \leq \frac{n}{2 \pi}+O(\sqrt{n})
$$

In order to obtain (1.1), Banaszczyk developed two measure inequalities (Lemma 1.5 of [3]) which have been crucial quantities in obtaining transference theorems for lattices and in the study of the computational complexity of lattice problems [1,2, 8, 10, 11. One of our results in this paper is to present an improvement of Lemma 1.5 of [3] with a concise yet elementary and transparent proof.

Using canonical norm inequalities, (1.1) can be generalized to a general convex body $U \in \mathfrak{C}_{n}$. Let $\mathbb{R}_{U}^{n}$ denote the space $\mathbb{R}^{n}$ equipped with norm $\|\cdot\|_{U}$. Then

$$
\xi(U) \leq \xi\left(\mathcal{B}_{2}^{n}\right) d\left(\mathbb{R}_{U}^{n}, \mathbb{R}_{2}^{n}\right) \leq\left(\frac{n}{2 \pi}+O(\sqrt{n})\right) n^{\frac{1}{2}} \leq C n^{\frac{3}{2}},
$$

where $d\left(\mathbb{R}_{U}^{n}, \mathbb{R}_{2}^{n}\right)$ denotes the Banach-Mazur distance between $\mathbb{R}_{U}^{n}$ and $\mathbb{R}_{2}^{n}$, and $C$ is a constant. By modifying the methods of [3], Banaszczyk [4,5] obtained a tighter bound $\xi(U) \leq C n \log n$ for $U \in \mathfrak{C}_{n}$ and, especially for $l_{p}$ norm, he proved

$$
\xi\left(\mathcal{B}_{p}^{n}\right) \leq C n \sqrt{\log n},(1 \leq p \leq \infty),
$$

where

$$
\mathcal{B}_{p}^{n}= \begin{cases}\left\{\mathbf{x} \in \mathbb{R}^{n}: \sum_{k=1}^{n}\left|x_{k}\right|^{p} \leq 1\right\}, & \text { if } 1 \leq p<\infty, \\ \left\{\mathbf{x} \in \mathbb{R}^{n}:\left|x_{k}\right| \leq 1 \text { for } k=1, \cdots, n\right\}, & \text { if } p=\infty\end{cases}
$$

is the closed unit ball in $l_{p}$ norm. More generally, for $U, V \in \mathfrak{C}_{n}$, [4] also considered the quantity

$$
\xi(U, V)=\sup _{L \in \mathcal{L}_{n}} \max _{1 \leq i \leq n} \lambda_{i}(L, U) \lambda_{n-i+1}\left(L^{*}, V\right)
$$

and proved that for some absolute constant $C$,

$(1.2) \xi\left(\mathcal{B}_{p}^{n}, \mathcal{B}_{q}^{n}\right) \leq C \sqrt{p q} n^{\frac{1}{p}+\frac{1}{q}},(1 \leq p, q<\infty, n=1,2, \cdots), \xi\left(\mathcal{B}_{\infty}^{n}, \mathcal{B}_{\infty}^{n}\right) \leq C \log n$.

Define $\nu_{n}(L, U)$ to be the smallest real number $r$ such that there are $n$ linearly independent lattice vectors in $r U$ that generate $L$. Similarly, define $\nu_{i}(L, U)$ to be the minimum $r$ such that there are $i$ linearly independent lattice vectors in $r U$ that can be extended to a basis of $L$. Obviously, $\lambda_{i}(L, U) \leq \nu_{i}(L, U), 1 \leq i \leq n$. In [7], Cai and Nerurkar proved that

$$
\forall n \geq 4, \quad \nu_{n}\left(L, \mathcal{B}_{2}^{n}\right) \leq \frac{\sqrt{n}}{2} \lambda_{n}\left(L, \mathcal{B}_{2}^{n}\right)
$$

Later in 6], Cai generalized the transference theorem to involve $\nu_{i}\left(L, \mathcal{B}_{2}^{n}\right)$ and obtained that for large enough $n$ and arbitrary lattice $L \in \mathcal{L}_{n}$, there exists some positive constant $C$ such that

$$
\lambda_{i}\left(L^{*}, \mathcal{B}_{2}^{n}\right) \nu_{n-i+1}\left(L, \mathcal{B}_{2}^{n}\right) \leq C n .
$$


Using canonical norm inequalities, the bound $C n^{\frac{3}{2}}$ can be achieved for a general convex body $U \in \mathfrak{C}_{n}$. It was remarked in [6] that it is possible to extend the results to include general norms. Actually our analysis shows that the upper bound in (1.3) may not hold for general norms. Our second contribution in this paper is to generalize (1.3) to general convex bodies and establish

$$
\lambda_{i}\left(L, \mathcal{B}_{p}^{n}\right) \nu_{n-i+1}\left(L^{*},\left(\mathcal{B}_{p}^{n}\right)^{\mathrm{o}}\right) \leq C n \sqrt{\log n}, 1 \leq p \leq \infty, n=1,2, \cdots .
$$

It is remarked that this result is better than that obtained by simply generalizing the inequality (1.3) using norm inequalities.

The rest of this paper is organized as follows: Section 2 reviews necessary concepts about the Gaussian measures on lattices and gives some useful lemmas. Our main results are stated and proved in section 3.

\section{Preliminaries}

2.1. Gaussian measures on lattices. The main tool of our proof is the Gaussian measure on lattices which was first proposed in [3] and further developed in [2, 8, 10]. For any $s>0$, define the Gaussian function centered at $\mathbf{c} \in \mathbb{R}^{n}$ with parameter $s$ as

$$
\forall \mathbf{x} \in \mathbb{R}^{n}, \quad \rho_{s, \mathbf{c}}(\mathbf{x})=\mathrm{e}^{-\pi\|\mathbf{x}-\mathbf{c}\|^{2} / s^{2}} .
$$

For any countable set $A \subset \mathbb{R}^{n}, \rho_{s, \mathbf{c}}(A)=\sum_{\mathbf{x} \in A} \rho_{s, \mathbf{c}}(\mathbf{x})$.

For any vector $\mathbf{c} \in \mathbb{R}^{n}$, real $s>0$, and lattice $L$, the discrete Gaussian distribution over $L$ is defined as

$$
\forall \mathbf{x} \in L, \quad D_{L, s, \mathbf{c}}(\mathbf{x})=\frac{\rho_{s, \mathbf{c}}(\mathbf{x})}{\rho_{s, \mathbf{c}}(L)} .
$$

When $s=1$ and/or $\mathbf{c}=\mathbf{0}$, the corresponding subscripts are omitted.

2.2. Fourier transform. For $\forall \mathbf{x}, \mathbf{y} \in \mathbb{R}^{n}$, the Fourier transform of a function $f: \mathbb{R}^{n} \rightarrow \mathbb{C}$ is defined to be

$$
\hat{f}(\mathbf{y})=\int_{\mathbb{R}^{n}} f(\mathbf{x}) \mathrm{e}^{-2 \pi \mathrm{i}\langle\mathbf{x}, \mathbf{y}\rangle} \mathrm{d} \mathbf{x} .
$$

We list several useful properties for our proof. If $f(\mathbf{x})=g(\mathbf{x}+\mathbf{v})$ for some function $g$ and vector $\mathbf{v}$, then $\hat{f}(\mathbf{y})=\mathrm{e}^{2 \pi \mathrm{i}\langle\mathbf{y}, \mathbf{v}\rangle} \hat{g}(\mathbf{y})$. Similarly, if $f(\mathbf{x})=\mathrm{e}^{2 \pi \mathrm{i}\langle\mathbf{x}, \mathbf{v}\rangle} g(\mathbf{x})$ for some function $g$ and vector $\mathbf{v}$, then $\hat{f}(\mathbf{y})=\hat{g}(\mathbf{y}-\mathbf{v})$. Particularly for the Gaussian function $\rho$, it is easy to deduce that $\hat{\rho}(\mathbf{y})=\rho(\mathbf{y}), \hat{\rho}_{s}(\mathbf{y})=s^{n} \rho_{1 / s}(\mathbf{y})$.

2.3. Useful lemmas. The following Poisson summation formula has been a useful tool in the theory of lattices. The proof of this formula can be found in [9].

Lemma 2.1 (8], Lemma 2.8). For a nice enough $f$ and any full-rank lattice $L$, $f(L)=\operatorname{det}\left(L^{*}\right) \hat{f}\left(L^{*}\right)$, where $\hat{f}$ is the Fourier transform of $f$.

Consider the Fourier transform of $D_{L}(x)$ :

$$
\hat{D}_{L}(\mathbf{y})=\int_{\mathbb{R}^{n}} \mathrm{e}^{-2 \pi \mathrm{i}\langle\mathbf{x}, \mathbf{y}\rangle} \mathrm{d} D_{L}(\mathbf{x})=\sum_{\mathbf{x} \in L} \mathrm{e}^{-2 \pi \mathrm{i}\langle\mathbf{x}, \mathbf{y}\rangle} D_{L}(\mathbf{x}) .
$$

Using Lemma 2.1, we get

$$
\sum_{\mathbf{x} \in L} \mathrm{e}^{-2 \pi \mathrm{i}\langle\mathbf{x}, \mathbf{y}\rangle} \frac{\rho(\mathbf{x})}{\rho(L)}=\frac{\operatorname{det}\left(L^{*}\right) \sum_{\mathbf{w} \in L^{*}} \rho(\mathbf{w}+\mathbf{y})}{\operatorname{det}\left(L^{*}\right) \rho\left(L^{*}\right)}=\frac{\rho\left(L^{*}+\mathbf{y}\right)}{\rho\left(L^{*}\right)} .
$$


Setting $\varphi_{L}(\mathbf{y})=\frac{\rho(L+\mathbf{y})}{\rho(L)}$, we have proved:

Lemma 2.2. For any $\mathbf{y} \in \mathbb{R}^{n}$ and lattice $L \in \mathcal{L}_{n}, \hat{D}_{L}(\mathbf{y})=\varphi_{L^{*}}(\mathbf{y})$.

Let $U$ be a convex body in $\mathbb{R}^{n}$ which is symmetric with respect to the origin. We denote

$$
\alpha(U)=\sup _{L \in \mathcal{L}_{n}} \frac{\rho(L \backslash U)}{\rho(L)}=\sup _{L \in \mathcal{L}_{n}} D_{L}(L \backslash U), \quad \beta(U)=\sup _{L \in \mathcal{L}_{n}} \sup _{\mathbf{u} \in \mathbb{R}^{n}} \frac{\rho((L+\mathbf{u}) \backslash U)}{\rho(L)} .
$$

Banaszczyk 4] gave the following measure inequalities:

Lemma 2.3 (4], Lemma 2.9). For arbitrary $r>0$ and $p \in[1, \infty)$, we have

$$
\beta\left(r \mathcal{B}_{p}^{n}\right)<p n \pi^{-\frac{p}{2}} \Gamma\left(\frac{p}{2}\right) r^{-p}, \quad \beta\left(r \mathcal{B}_{\infty}^{n}\right)<2 n \mathrm{e}^{-\pi r^{2}} .
$$

The above lemma generalizes the following lemma of $[3]$.

Lemma 2.4 ([3], Lemma 1.5). For any $c>\frac{1}{\sqrt{2 \pi}}$,

$$
\alpha\left(c \sqrt{n} \mathcal{B}_{2}^{n}\right)<C^{n}, \quad \beta\left(c \sqrt{n} \mathcal{B}_{2}^{n}\right)<2 C^{n},
$$

where $C=c \sqrt{2 \pi \mathrm{e}} \cdot \mathrm{e}^{-\pi c^{2}}<1$.

This lemma is the key in the proof of the transference theorem and plays a significant role in applying Gaussian measures in lattice-based cryptography.

\section{MAIN RESUltS}

In this section, we shall discuss our two main results. In section 3.1, we present a simplified proof of an improvement of Lemma 2.4. Section 3.2 generalizes Cai's transference theorem (namely inequality (1.3)) to general convex bodies.

3.1. A simple derivation of Lemma 2.4. In an effort to improve Lemma 2.4. we are able to get a better bound for $\beta(U)$ by improving Banaszczyk's bound by a factor of 2. Meanwhile, our proof for $\alpha(U)$ and $\beta(U)$ is uniform and concise, yet elementary and transparent without involving moment estimation, derivative calculation, and other complex derivations.

Theorem 3.1. For any constant $c>1 / \sqrt{2 \pi}$, we have

$$
\alpha\left(c \sqrt{n} \mathcal{B}_{2}^{n}\right)<C^{n}, \quad \beta\left(c \sqrt{n} \mathcal{B}_{2}^{n}\right)<C^{n},
$$

where $C=c \sqrt{2 \pi \mathrm{e}} \cdot \mathrm{e}^{-\pi c^{2}}<1$.

Proof. By the definition of $\alpha(U)$ and $\beta(U)$, it is enough to prove $\beta\left(c \sqrt{n} \mathcal{B}_{2}^{n}\right)<C^{n}$. Let $s=c \sqrt{2 \pi}>1$. For any $L \in \mathcal{L}_{n}, \mathbf{v} \in \mathbb{R}^{n}$, we have

$$
\begin{aligned}
\rho_{s}(L+\mathbf{v}) & =\operatorname{det}\left(L^{*}\right) s^{n} \sum_{\mathbf{y} \in L^{*}} \rho_{\frac{1}{s}}(\mathbf{y}) \mathrm{e}^{2 \pi \mathrm{i}\langle\mathbf{y}, \mathbf{v}\rangle} \leq \operatorname{det}\left(L^{*}\right) s^{n} \sum_{\mathbf{y} \in L^{*}} \rho_{\frac{1}{s}}(\mathbf{y}) \\
& \leq \operatorname{det}\left(L^{*}\right) s^{n} \sum_{\mathbf{y} \in L^{*}} \rho(\mathbf{y})=s^{n} \rho(L) .
\end{aligned}
$$


On the other hand,

$$
\begin{aligned}
& \rho_{s}(L+\mathbf{v})>\rho_{s}\left((L+\mathbf{v}) \backslash c \sqrt{n} \mathcal{B}_{2}^{n}\right)=\sum_{\mathbf{y} \in L+\mathbf{v},\|\mathbf{y}\| \geq c \sqrt{n}} \mathrm{e}^{-\pi\left\|\frac{\mathbf{y}}{s}\right\|^{2}} \\
& =\sum_{\mathbf{y} \in L+\mathbf{v},\|\mathbf{y}\| \geq c \sqrt{n}} \mathrm{e}^{-\pi\|\mathbf{y}\|^{2}} \mathrm{e}^{\pi\left(1-\frac{1}{s^{2}}\right)\|\mathbf{y}\|^{2}} \geq \mathrm{e}^{\pi\left(1-\frac{1}{s^{2}}\right) c^{2} n} \sum_{\mathbf{y} \in L+\mathbf{v},\|\mathbf{y}\| \geq c \sqrt{n}} \mathrm{e}^{-\pi\|\mathbf{y}\|^{2}} \\
& =\mathrm{e}^{\pi\left(1-\frac{1}{s^{2}}\right) c^{2} n} \rho\left((L+\mathbf{v}) \backslash c \sqrt{n} \mathcal{B}_{2}^{n}\right) .
\end{aligned}
$$

Hence $\mathrm{e}^{\pi\left(1-\frac{1}{s^{2}}\right) c^{2} n} \rho((L+\mathbf{v}) \backslash c \sqrt{n} \mathcal{B})<s^{n} \rho(L)$, which implies that

$$
\rho((L+\mathbf{v}) \backslash c \sqrt{n} \mathcal{B})<\left(\frac{s}{\mathrm{e}^{\pi\left(1-\frac{1}{s^{2}}\right) c^{2}}}\right)^{n} \rho(L)=C^{n} \rho(L) .
$$

Remark 3.2. Generally, for any $L \in \mathcal{L}_{n}, \mathbf{v} \in \mathbb{R}^{n}$ and any constant $c>1 / \sqrt{2 \pi}$, we have

$$
\frac{\rho_{s}\left((L+\mathbf{v}) \backslash c \cdot s \cdot \sqrt{n} \mathcal{B}_{2}^{n}\right)}{\rho_{s}(L)}<C^{n},
$$

where $C=c \sqrt{2 \pi \mathrm{e}} \cdot \mathrm{e}^{-\pi c^{2}}$ is the same as that in Theorem 3.1

Remark 3.3. Using the same method and norm inequalities, we can prove that

(1) If $p \in[1,2]$, then for any $L \in \mathcal{L}_{n}, \mathbf{v} \in \mathbb{R}^{n}$ and any $r>\frac{1}{\sqrt{2 \pi}} n^{\frac{1}{p}}$, we have

$$
\frac{\rho\left((L+\mathbf{v}) \backslash r \mathcal{B}_{p}^{n}\right)}{\rho(L)} \leq C^{n},
$$

where $C=r n^{-\frac{1}{p}} \sqrt{2 \pi \mathrm{e}} \cdot \mathrm{e}^{-\pi r^{2} n^{-\frac{2}{p}}}<1$. The upper bound is tighter than that in Lemma 2.3 when $r>\frac{1}{\sqrt{2 \pi}} n^{\frac{1}{p}}$.

(2) If $p \in[2,+\infty)$, then for any $L \in \mathcal{L}_{n}, \mathbf{v} \in \mathbb{R}^{n}$ and any $r>\frac{1}{\sqrt{2 \pi}} n^{\frac{1}{2}}$, we have

$$
\frac{\rho\left((L+\mathbf{v}) \backslash r \mathcal{B}_{p}^{n}\right)}{\rho(L)} \leq C^{n},
$$

where $C=r n^{-\frac{1}{2}} \sqrt{2 \pi \mathrm{e}} \cdot \mathrm{e}^{-\pi r^{2} n^{-1}}<1$. The upper bound is tighter than that in Lemma 2.3 when $r>\frac{1}{\sqrt{2 \pi}} n^{\frac{1}{2}}$.

3.2. New transference theorem for general convex bodies. In this part, we generalize Cai's transference theorem (namely the inequality (1.3)) to general convex bodies. We start with the following key technical lemma.

Lemma 3.4. For any lattice $L \in \mathcal{L}_{n}$, if $U, V \in \mathfrak{C}_{n}$ and $2 \alpha(U)+\beta(V) \leq 1$, then

$$
\nu_{n-i+1}(L, U) \lambda_{i}\left(L^{*}, V\right) \leq 3 .
$$

The proof of Lemma 3.4 is a slight modification of the proof of Theorem 4.1 in [6]. In order for the paper to be self-contained, we give a sketch of the proof.

Sketch of the proof. Suppose that $\nu_{n-i+1}(L, U) \lambda_{i}\left(L^{*}, V\right)>3$. Then there exists some lattice $L \in \mathcal{L}_{n}$ with $\nu_{n-i+1}(L, U)>1$ and $\lambda_{i}\left(L^{*}, V\right)>3$.

By the definition of $\lambda_{j}\left(L^{*}, V\right)$, we can find linearly independent lattice vectors $\mathbf{s}_{1}, \cdots, \mathbf{s}_{i-1} \in L^{*}$ such that $\left\|\mathbf{s}_{j}\right\|_{V}=\lambda_{j}\left(L^{*}, V\right), j=1, \cdots, i-1$. Let $S=$ $\operatorname{span}\left\{\mathbf{s}_{1}, \cdots, \mathbf{s}_{i-1}\right\}$; then $\operatorname{dim} S=i-1$ and $\forall \mathbf{u} \in L^{*} \backslash S,\|\mathbf{u}\|_{V} \geq \lambda_{i}\left(L^{*}, V\right)$. 
Let $\mathbf{b}_{1}^{*}, \cdots, \mathbf{b}_{i-1}^{*}$ be a basis of $L^{*} \cap S$, and define the orthogonal projection $P: \operatorname{span}\left\{L^{*}\right\} \mapsto \operatorname{span}\left\{\mathbf{b}_{1}^{*}, \cdots, \mathbf{b}_{i-1}^{*}\right\}^{\perp}, \quad \forall \mathbf{b} \in \operatorname{span}\left\{L^{*}\right\}, P(\mathbf{b})=\mathbf{b}-\sum_{j=1}^{i-1} \frac{\left\langle\mathbf{b}, \tilde{\mathbf{b}}_{j}^{*}\right\rangle}{\left\langle\tilde{\mathbf{b}}_{j}^{*}, \tilde{\mathbf{b}}_{j}^{*}\right\rangle} \cdot \tilde{\mathbf{b}}_{j}^{*}$,

where $\tilde{\mathbf{b}}_{j}^{*}$ is the Gram-Schmidt orthogonal vector of $\mathbf{b}_{j}^{*}(j=1, \cdots, i-1)$. Then $P\left(L^{*}\right)$ is a lattice of dimension $n-i+1$. Assume that $P\left(\mathbf{b}_{i}^{*}\right), \cdots, P\left(\mathbf{b}_{n}^{*}\right)$ with $\mathbf{b}_{i}^{*}, \cdots$, $\mathbf{b}_{n}^{*} \in L^{*}$ is a basis of $P\left(L^{*}\right)$. Then it is easy to verify that $\mathbf{b}_{1}^{*}, \cdots, \mathbf{b}_{i-1}^{*}, \mathbf{b}_{i}^{*}, \cdots$, $\mathbf{b}_{n}^{*}$ is a basis of the lattice $L^{*}$. Let $\mathbf{b}_{1}, \cdots, \mathbf{b}_{i-1}, \mathbf{b}_{i}, \cdots, \mathbf{b}_{n}$ be its dual basis for $L$. Then

$$
S=\operatorname{span}\left\{\mathbf{s}_{1}, \cdots, \mathbf{s}_{i-1}\right\}=\operatorname{span}\left\{\mathbf{b}_{1}^{*}, \cdots, \mathbf{b}_{i-1}^{*}\right\}, \quad L \cap S^{\perp}=L\left(\mathbf{b}_{i}, \cdots, \mathbf{b}_{n}\right),
$$

where $L\left(\mathbf{b}_{i}, \cdots, \mathbf{b}_{n}\right)$ denotes the lattice generated by the basis $\mathbf{b}_{i}, \cdots, \mathbf{b}_{n}$. Following Cai's route in [6], we define the orthogonal projection from $\mathbb{R}^{n}$ to $S^{\perp}$ :

$$
\pi: \mathbb{R}^{n} \rightarrow S^{\perp}, \quad \sum_{j=1}^{i-1} x_{j} \mathbf{b}_{j}^{*}+\sum_{j=i}^{n} x_{j} \mathbf{b}_{j} \mapsto \sum_{j=i}^{n} x_{j} \mathbf{b}_{j}
$$

and the projection

$$
\varphi: \mathbb{R}^{n} \rightarrow S^{\perp}, \quad \sum_{j=1}^{n} x_{j} \mathbf{b}_{j} \mapsto \sum_{j=i}^{n} x_{j} \mathbf{b}_{j}
$$

Obviously,

$$
\varphi(L)=L \cap S^{\perp}=\left\{\sum_{j=i}^{n} x_{j} \mathbf{b}_{j}: x_{j} \in \mathbb{Z}\right\} .
$$

Moreover, $\operatorname{dim}\left(\operatorname{span}\left\{\pi\left(L^{*}\right)\right\}\right)=\operatorname{dim}\left(S^{\perp}\right)=n-i+1$ and $\pi\left(L^{*}\right) \subseteq S^{\perp}$; hence $\operatorname{span}\left\{\pi\left(L^{*}\right)\right\}=S^{\perp}$. Meanwhile, due to the fact that $\operatorname{ker}(\pi)=S, \pi\left(L^{*}\right)$ is a lattice generated by the basis $\pi\left(\mathbf{b}_{i}^{*}\right), \cdots, \pi\left(\mathbf{b}_{n}^{*}\right)$. From the fact that $\forall j, k \geq i,\left\langle\mathbf{b}_{j}, \pi\left(\mathbf{b}_{k}^{*}\right)\right\rangle=$ $\left\langle\mathbf{b}_{j}, \mathbf{b}_{k}^{*}\right\rangle=\delta_{j k}$ and the definition of dual lattice, we can easily deduce that

$$
\pi\left(L^{*}\right)=\left(L \cap S^{\perp}\right)^{*} .
$$

In fact, the above analysis gives an alternative and succinct proof of Lemma 4.1 of [6].

Let $L_{1}$ be the sublattice of $L$ generated by all lattice vectors in $L \cap U$. Since $\nu_{n-i+1}(L, U)>1, L_{1}$ does not contain any $(n-i+1)$ linearly independent lattice vectors that can be extended to be a basis of $L$. Obviously, $\varphi\left(L_{1}\right) \subseteq \varphi(L)=$ $L \cap S^{\perp}$. In fact, it is easy to verify that $\varphi\left(L_{1}\right)$ is a proper sublattice of $L \cap S^{\perp}$. If $\operatorname{dim}\left(\varphi\left(L_{1}\right)\right)=n-i+1$, then let $\tilde{L}=\varphi\left(L_{1}\right)$; otherwise extend $\varphi\left(L_{1}\right)$ to be a $(n-i+1)$-dimensional proper sublattice $\tilde{L}$ of $L \cap S^{\perp}$ which contains $\varphi\left(L_{1}\right)$. Now let

$$
L_{2}=\tilde{L} \oplus\left\langle\mathbf{b}_{1}\right\rangle \oplus \cdots \oplus\left\langle\mathbf{b}_{i-1}\right\rangle .
$$

Then it is easy to verify that $L_{1} \subseteq L_{2}, \operatorname{dim}\left(L_{2}\right)=n$ and $L_{2}$ is a proper sublattice of $L$.

Lemma 3.5. There exists a vector $\mathbf{x} \in\left(L_{2}\right)^{*}$ such that

$$
\operatorname{dist}_{V}\left(\mathbf{x}, L^{*}\right)=\min _{\mathbf{y} \in L^{*}}\|\mathbf{x}-\mathbf{y}\|_{V} \geq \frac{\lambda_{i}\left(L^{*}, V\right)}{3} .
$$


The proof of Lemma 3.5 is some combination of the proofs of Lemma 4.5 and Lemma 4.6 in [6], but our metric is induced by the general convex body $V$. For completeness, we provide the proof of this lemma in the appendix.

Now, let $\mathbf{u}$ be the $\mathbf{x}$ promised in Lemma 3.5. Then from the evenness of the function $D_{L}$, we have

$$
\begin{aligned}
\hat{D}_{L}(\mathbf{u})= & \sum_{\mathbf{v} \in L} D_{L}(\mathbf{v}) \mathrm{e}^{-2 \pi \mathrm{i}\langle\mathbf{u}, \mathbf{v}\rangle}=\sum_{\mathbf{v} \in L} D_{L}(\mathbf{v}) \cos 2 \pi\langle\mathbf{u}, \mathbf{v}\rangle \\
= & \sum_{\mathbf{v} \in L \backslash L_{2}} D_{L}(\mathbf{v}) \cos 2 \pi\langle\mathbf{u}, \mathbf{v}\rangle+\sum_{\mathbf{v} \in L_{2}} D_{L}(\mathbf{v}) \cos 2 \pi\langle\mathbf{u}, \mathbf{v}\rangle \\
= & \sum_{\mathbf{v} \in L \backslash L_{2}} D_{L}(\mathbf{v}) \cos 2 \pi\langle\mathbf{u}, \mathbf{v}\rangle+\sum_{\mathbf{v} \in L_{2}} D_{L_{2}}(\mathbf{v}) \cos 2 \pi\langle\mathbf{u}, \mathbf{v}\rangle \\
& +\sum_{\mathbf{v} \in L_{2}}\left(D_{L}(\mathbf{v})-D_{L_{2}}(\mathbf{v})\right) \cos 2 \pi\langle\mathbf{u}, \mathbf{v}\rangle \\
= & \hat{D}_{L_{2}}(\mathbf{u})+\sum_{\mathbf{v} \in L \backslash L_{2}} D_{L}(\mathbf{v}) \cos 2 \pi\langle\mathbf{u}, \mathbf{v}\rangle+\sum_{\mathbf{v} \in L_{2}}\left(D_{L}(\mathbf{v})-D_{L_{2}}(\mathbf{v})\right) \cos 2 \pi\langle\mathbf{u}, \mathbf{v}\rangle .
\end{aligned}
$$

The facts

$$
\left|\sum_{\mathbf{v} \in L \backslash L_{2}} D_{L}(\mathbf{v}) \cos 2 \pi\langle\mathbf{u}, \mathbf{v}\rangle\right|<\sum_{\mathbf{v} \in L \backslash U} D_{L}(\mathbf{v})=D_{L}(L \backslash U) \leq \alpha(U)
$$

and

$$
\begin{aligned}
\left|\sum_{\mathbf{v} \in L_{2}}\left(D_{L}(\mathbf{v})-D_{L_{2}}(\mathbf{v})\right) \cos 2 \pi\langle\mathbf{u}, \mathbf{v}\rangle\right| & \leq \sum_{\mathbf{v} \in L_{2}}\left|D_{L}(\{\mathbf{v}\})-D_{L_{2}}(\{\mathbf{v}\})\right| \\
& =\sum_{\mathbf{v} \in L_{2}}\left|\frac{\rho(\mathbf{v})}{\rho(L)}-\frac{\rho(\mathbf{v})}{\rho\left(L_{2}\right)}\right|=\frac{\rho\left(L \backslash L_{2}\right)}{\rho(L) \rho\left(L_{2}\right)} \sum_{\mathbf{v} \in L_{2}} \rho(\mathbf{v}) \\
& =\frac{\rho\left(L \backslash L_{2}\right)}{\rho(L)} \leq \frac{\rho(L \backslash U)}{\rho(L)} \leq \alpha(U)
\end{aligned}
$$

yield

$$
\hat{D}_{L}(\mathbf{u})>\hat{D}_{L_{2}}(\mathbf{u})-2 \alpha(U) .
$$

Since $\left(L_{2}\right)^{*}+\mathbf{u}=\left(L_{2}\right)^{*}$, from Lemma 2.2 we see that $\hat{D}_{L_{2}}(\mathbf{u})=\varphi_{\left(L_{2}\right)^{*}}(\mathbf{u})=$ $\frac{\rho\left(\left(L_{2}\right)^{*}+\mathbf{u}\right)}{\rho\left(\left(L_{2}\right)^{*}\right)}=1$. Hence

$$
\hat{D}_{L}(\mathbf{u})>1-2 \alpha(U) \text {. }
$$

On the other hand,

$$
\operatorname{dist}_{V}\left(\mathbf{u}, L^{*}\right)=\min _{\mathbf{y} \in L^{*}}\|\mathbf{u}-\mathbf{y}\|_{V} \geq \frac{\lambda_{i}\left(L^{*}, V\right)}{3}>1
$$

implies $\left(L^{*}+\mathbf{u}\right) \cap V=\emptyset$. From Lemma 2.2 again,

$$
\hat{D}_{L}(\mathbf{u})=\varphi_{L^{*}}(\mathbf{u})=\frac{\rho\left(L^{*}+\mathbf{u}\right)}{\rho\left(L^{*}\right)}=\frac{\rho\left(\left(L^{*}+\mathbf{u}\right) \backslash V\right)}{\rho\left(L^{*}\right)} \leq \beta(V) .
$$

Combining (3.2) and (3.3), we get $2 \alpha(U)+\beta(V)>1$. This is a contradiction. 
Based on Lemma 3.4, we can deduce our second main result.

Theorem 3.6. For any lattice $L \in \mathcal{L}_{n}, 1 \leq p, q<\infty, i=1,2, \cdots, n$, there exist three positive constants $C_{1}, C_{2}, C_{3}$ such that

$$
\begin{gathered}
\lambda_{i}\left(L, \mathcal{B}_{p}^{n}\right) \nu_{n-i+1}\left(L^{*}, \mathcal{B}_{q}^{n}\right) \leq C_{1} \sqrt{p q} n^{\frac{1}{p}+\frac{1}{q}}, \\
\lambda_{i}\left(L, \mathcal{B}_{p}^{n}\right) \nu_{n-i+1}\left(L^{*}, \mathcal{B}_{\infty}^{n}\right) \leq C_{2} \sqrt{p} n^{\frac{1}{p}}(\log n)^{\frac{1}{2}}, \\
\lambda_{i}\left(L, \mathcal{B}_{\infty}^{n}\right) \nu_{n-i+1}\left(L^{*}, \mathcal{B}_{p}^{n}\right) \leq C_{2} \sqrt{p} n^{\frac{1}{p}}(\log n)^{\frac{1}{2}}, \\
\lambda_{i}\left(L, \mathcal{B}_{\infty}^{n}\right) \nu_{n-i+1}\left(L^{*}, \mathcal{B}_{\infty}^{n}\right) \leq C_{3} \log n .
\end{gathered}
$$

Proof. Combining Lemma 2.3 with the basic property of the gamma function, we have

$$
\alpha\left(r \mathcal{B}_{p}^{n}\right) \leq \beta\left(r \mathcal{B}_{p}^{n}\right)<p n \pi^{-\frac{p}{2}} \Gamma\left(\frac{p}{2}\right) r^{-p}=2 n \pi^{-\frac{p}{2}} \Gamma\left(\frac{p}{2}+1\right) r^{-p}
$$

Let

$$
r_{1}=\left(c_{1} n \pi^{-\frac{p}{2}} \Gamma\left(\frac{p}{2}+1\right)\right)^{\frac{1}{p}}=c_{p} n^{\frac{1}{p}} \sqrt{p}, \quad r_{2}=\left(c_{2} n \pi^{-\frac{p}{2}} \Gamma\left(\frac{p}{2}+1\right)\right)^{\frac{1}{q}}=c_{q} n^{\frac{1}{q}} \sqrt{q}
$$

where $c_{1}, c_{2}$ are sufficiently large constants such that $2 \alpha\left(r_{1} \mathcal{B}_{p}^{n}\right)+\beta\left(r_{2} \mathcal{B}_{q}^{n}\right) \leq 1$. Hence

$$
\begin{aligned}
\lambda_{i}\left(L, \mathcal{B}_{p}^{n}\right) \nu_{n-i+1}\left(L^{*}, \mathcal{B}_{q}^{n}\right) & =r_{1} r_{2} \lambda_{i}\left(L, r_{1} \mathcal{B}_{p}^{n}\right) \nu_{n-i+1}\left(L^{*}, r_{2} \mathcal{B}_{q}^{n}\right) \\
& \leq 3 r_{1} r_{2}=C_{1} \sqrt{p q} n^{\frac{1}{p}+\frac{1}{q}}
\end{aligned}
$$

Similarly, we can prove the inequalities (3.5)-(3.7).

Since $\left(\mathcal{B}_{p}^{n}\right)^{\mathrm{o}}=\mathcal{B}_{q}^{n}$, where $\frac{1}{p}+\frac{1}{q}=1$, we have the following.

Corollary 3.7. For any lattice $L \in \mathcal{L}_{n}$, there exists a positive constant $C$ such that

$$
\lambda_{i}\left(L, \mathcal{B}_{p}^{n}\right) \nu_{n-i+1}\left(L^{*},\left(\mathcal{B}_{p}^{n}\right)^{\mathrm{o}}\right) \leq C n \sqrt{\frac{p^{2}}{p-1}}, 1<p<\infty, i=1,2, \cdots, n .
$$

Remark 3.8. Compared with Babaszczyk's result (inequality (1.2)) which implies that for some absolute constant $C^{\prime}>0$,

$$
\lambda_{i}\left(L, \mathcal{B}_{p}^{n}\right) \lambda_{n-i+1}\left(L^{*},\left(\mathcal{B}_{p}^{n}\right)^{\circ}\right) \leq C^{\prime} n \sqrt{\frac{p^{2}}{p-1}}, 1<p<\infty, i=1,2, \cdots, n,
$$

Corollary 3.7 yields an improvement by replacing the successive minima $\lambda_{n-i+1}$ with $\nu_{n-i+1}$. 
Corollary 3.9. For any lattice $L \in \mathcal{L}_{n}$, there exists a positive constant $C$ such that

$$
\lambda_{i}\left(L, \mathcal{B}_{p}^{n}\right) \nu_{n-i+1}\left(L^{*},\left(\mathcal{B}_{p}^{n}\right)^{\circ}\right) \leq C n \sqrt{\log n}, 1 \leq p \leq \infty, i=1,2, \cdots, n .
$$

Proof. For arbitrary $p \in[1, \infty]$, let $q=\frac{p}{p-1}$; then $\left(\mathcal{B}_{p}^{n}\right)^{\circ}=\mathcal{B}_{q}^{n}$. Without loss of generality, we assume that $p \leq 2 \leq q$. Since

$$
\|\mathbf{x}\|_{2} \leq\|\mathbf{x}\|_{p} \leq n^{\frac{1}{p}-\frac{1}{2}}\|\mathbf{x}\|_{2}, \quad\|\mathbf{x}\|_{q} \leq n^{\frac{1}{q}}\|\mathbf{x}\|_{\infty} .
$$

Let $r=n^{\frac{1}{2}-\frac{1}{p}}$ and $s=n^{-\frac{1}{q}}$; then $r \mathcal{B}_{2}^{n} \subset \mathcal{B}_{p}^{n}$ and $s \mathcal{B}_{\infty}^{n} \subset \mathcal{B}_{q}^{n}$. Thus from Theorem 3.6. we have

$$
\begin{aligned}
\lambda_{i}(L, & \left.\mathcal{B}_{p}^{n}\right) \nu_{n-i+1}\left(L^{*},\left(\mathcal{B}_{p}^{n}\right)^{\mathrm{o}}\right) \\
& =\lambda_{i}\left(L, \mathcal{B}_{p}^{n}\right) \nu_{n-i+1}\left(L^{*}, \mathcal{B}_{q}^{n}\right) \leq \lambda_{i}\left(L, r \mathcal{B}_{2}^{n}\right) \nu_{n-i+1}\left(L^{*}, s \mathcal{B}_{\infty}^{n}\right) \\
& =\frac{1}{r s} \lambda_{i}\left(L, \mathcal{B}_{2}^{n}\right) \nu_{n-i+1}\left(L^{*}, \mathcal{B}_{\infty}^{n}\right) \leq C n^{\frac{1}{2}}(\log n)^{\frac{1}{2}} n^{\frac{1}{p}-\frac{1}{2}} n^{\frac{1}{q}}=C n \sqrt{\log n} .
\end{aligned}
$$

Remark 3.10. In [5], Banaszczyk pointed out that there exists a lattice $L \in \mathcal{L}_{n}$ such that

$$
\lambda_{i}\left(L, \mathcal{B}_{p}^{n}\right) \lambda_{n-i+1}\left(L^{*},\left(\mathcal{B}_{p}^{n}\right)^{\mathrm{o}}\right) \geq \tilde{C} n
$$

for some positive constant $\tilde{C}$. Therefore, for this lattice,

$$
\lambda_{i}\left(L, \mathcal{B}_{p}^{n}\right) \nu_{n-i+1}\left(L^{*},\left(\mathcal{B}_{p}^{n}\right)^{\circ}\right) \geq \lambda_{i}\left(L, \mathcal{B}_{p}^{n}\right) \lambda_{n-i+1}\left(L^{*},\left(\mathcal{B}_{p}^{n}\right)^{\circ}\right) \geq \tilde{C} n .
$$

Hence the upper bound of Corollary 3.9 is close to optimal with deviation at most a factor of $\sqrt{\log n}$.

\section{APPENDIX}

Proof of Lemma 3.5. First we show that there exists a vector $\mathbf{v} \in\left(L_{2}\right)^{*} \backslash\left(L^{*}+S\right)$. Combining the equality (1.3) with $\tilde{L} \subset L \cap S^{\perp}$, we have $\tilde{L}^{*} \supset\left(L \cap S^{\perp}\right)^{*}=\pi\left(L^{*}\right)$. Then there exists a vector $\mathbf{w} \in(\tilde{L})^{*} \backslash \pi\left(L^{*}\right)$. Let $\mathbf{v}=\mathbf{w}-\sum_{k=1}^{i-1}\left\langle\mathbf{w}, \mathbf{b}_{k}\right\rangle \mathbf{b}_{k}^{*}$. Since $\mathbf{w} \in S^{\perp}$ and $\sum_{k=1}^{i-1}\left\langle\mathbf{w}, \mathbf{b}_{k}\right\rangle \mathbf{b}_{k}^{*} \in S, \pi(\mathbf{v})=\mathbf{w}$. This indicates $\mathbf{v} \notin L^{*}+S$. Moreover, for any $\mathbf{t} \in L_{2}, \mathbf{t}=\varphi(\mathbf{t})+\sum_{l=1}^{i-1} z_{l} \mathbf{b}_{l}$, where $z_{l} \in \mathbb{Z}$. Since $\varphi(\mathbf{t}) \in \tilde{L} \subset L \cap S^{\perp}$, we have

$$
\begin{aligned}
\langle\mathbf{v}, \mathbf{t}\rangle= & \left\langle\mathbf{w}-\sum_{k=1}^{i-1}\left\langle\mathbf{w}, \mathbf{b}_{k}\right\rangle \mathbf{b}_{k}^{*}, \varphi(\mathbf{t})+\sum_{l=1}^{i-1} z_{l} \mathbf{b}_{l}\right\rangle \\
= & \langle\mathbf{w}, \varphi(\mathbf{t})\rangle-\left\langle\sum_{k=1}^{i-1}\left\langle\mathbf{w}, \mathbf{b}_{k}\right\rangle \mathbf{b}_{k}^{*}, \varphi(\mathbf{t})\right\rangle+\left\langle\mathbf{w}, \sum_{l=1}^{i-1} z_{l} \mathbf{b}_{l}\right\rangle \\
& -\left\langle\sum_{k=1}^{i-1}\left\langle\mathbf{w}, \mathbf{b}_{k}\right\rangle \mathbf{b}_{k}^{*}, \sum_{l=1}^{i-1} z_{l} \mathbf{b}_{l}\right\rangle \\
= & \langle\mathbf{w}, \varphi(\mathbf{t})\rangle-0+\sum_{l=1}^{i-1} z_{l}\left\langle\mathbf{w}, \mathbf{b}_{l}\right\rangle-\sum_{l=1}^{i-1} z_{l}\left\langle\mathbf{w}, \mathbf{b}_{l}\right\rangle=\langle\mathbf{w}, \varphi(\mathbf{t})\rangle \in \mathbb{Z} .
\end{aligned}
$$

This implies that $\mathbf{v} \in\left(L_{2}\right)^{*}$. 
Now we prove the result by contradiction. Assume that for any $\mathbf{x} \in\left(L_{2}\right)^{*}$, there exists a $\mathbf{y} \in L^{*}$ such that $\|\mathbf{x}-\mathbf{y}\|_{V}<\frac{\lambda_{i}\left(L^{*}, V\right)}{3}$. Specifically, choose $\mathbf{x} \in$ $\left(L_{2}\right)^{*} \backslash\left(L^{*}+S\right)$ and let $\mathbf{s}=\mathbf{x}-\mathbf{y}$; then $\mathbf{s} \in\left(L_{2}\right)^{*} \backslash\left(L^{*}+S\right)$ is a nonzero vector.

Consider the lattice points in the set $\{k \mathbf{s}: k \in \mathbb{Z}$ and $k \geq 1\}$. By assumption, for each $k \mathbf{s}$, there exists a point in $L^{*}$ with distance less than $\frac{\lambda_{i}\left(L^{*}, V\right)}{3}$. By definition, $\mathbf{s}$ is associated to $\mathbf{0} \in L^{*} \cap S$. But it is clear that $k \mathbf{s}$ cannot be associated to a point in $L^{*} \cap S$ if $k$ is large. Let $k_{0}$ be the first $k$ such that $k \mathbf{s}$ is associated with a $\mathbf{z} \in L^{*} \backslash S$, and let $\mathbf{z}_{0} \in L^{*} \cap S$ be the point such that $\left\|\mathbf{z}_{0}-\left(k_{0}-1\right) \mathbf{s}\right\|_{V}<\frac{\lambda_{i}\left(L^{*}, V\right)}{3}$. Then

$$
\begin{aligned}
\left\|\mathbf{z}-\mathbf{z}_{0}\right\|_{V} & =\left\|\mathbf{z}-k_{0} \mathbf{s}+\mathbf{s}+\left(k_{0}-1\right) \mathbf{s}-\mathbf{z}_{0}\right\|_{V} \\
& <\frac{\lambda_{i}\left(L^{*}, V\right)}{3}+\frac{\lambda_{i}\left(L^{*}, V\right)}{3}+\frac{\lambda_{i}\left(L^{*}, V\right)}{3}=\lambda_{i}\left(L^{*}, V\right) .
\end{aligned}
$$

This is a contradiction, and the lemma is proved.

\section{ACKNOWLEDGEMENTS}

The authors thank the anonymous referees for their thorough and useful comments which helped to improve the presentation of this paper.

\section{REFERENCES}

[1] Dorit Aharonov and Oded Regev. A lattice problem in quantum NP. In Proceedings of FOCS 2003, pages 210-219.

[2] Dorit Aharonov and Oded Regev, Lattice problems in NP $\cap$ coNP, J. ACM 52 (2005), no. 5, 749-765 (electronic), DOI 10.1145/1089023.1089025. MR.2176561 (2006k:68046)

[3] W. Banaszczyk, New bounds in some transference theorems in the geometry of numbers, Math. Ann. 296 (1993), no. 4, 625-635, DOI 10.1007/BF01445125. MR1233487 (94k:11075)

[4] W. Banaszczyk, Inequalities for convex bodies and polar reciprocal lattices in $\mathbf{R}^{n}$, Discrete Comput. Geom. 13 (1995), no. 2, 217-231, DOI 10.1007/BF02574039. MR.1314964 (95m:11068)

[5] W. Banaszczyk, Inequalities for convex bodies and polar reciprocal lattices in $\mathbf{R}^{n}$. II. Application of K-convexity, Discrete Comput. Geom. 16 (1996), no. 3, 305-311, DOI 10.1007/BF02711514. MR1410163 (97h:11073)

[6] Jin-Yi Cai, A new transference theorem in the geometry of numbers and new bounds for $A j$ tai's connection factor, Fifth Annual International Computing and Combinatorics Conference (COCOON'99) (Tokyo), Discrete Appl. Math. 126 (2003), no. 1, 9-31, DOI 10.1016/S0166218X(02)00216-0. MR1955767 (2005a:11100)

[7] Jin-yi Cai, Ajay Nerurkar. An Improved Worst-Case to Average-Case Reduction for Lattice Problems. In Proceedings of FOCS 1997, pp. 468-477.

[8] Daniele Micciancio and Oded Regev, Worst-case to average-case reductions based on Gaussian measures, SIAM J. Comput. 37 (2007), no. 1, 267-302 (electronic), DOI 10.1137/S0097539705447360. MR2306293 (2008c:68037)

[9] Wolfgang Ebeling, Lattices and codes, A course partially based on lectures by F. Hirzebruch, Second revised edition, Advanced Lectures in Mathematics, Friedr. Vieweg \& Sohn, Braunschweig, 2002. MR.1938666(2003i:11093)

[10] Oded Regev, New lattice-based cryptographic constructions, J. ACM 51 (2004), no. 6, 899-942 (electronic), DOI 10.1145/1039488.1039490. MR2145258(2006e:94044)

[11] Oded Regev, On lattices, learning with errors, random linear codes, and cryptography, STOC'05: Proceedings of the 37th Annual ACM Symposium on Theory of Computing, ACM, New York, 2005, pp. 84-93, DOI 10.1145/1060590.1060603. MR2181605 (2006g:94031) 
Key Lab of Cryptologic Technology and Information Security, Ministry of Education, Shandong University, Jinan, 250100, People's Republic of China - And - School of Mathematics, Shandong University, Jinan, 250100, People's Republic of China

E-mail address: chengliangtian@mail.sdu.edu.cn

Current address: SKLOIS, Institute of Information Engineering, Chinese Academy of Sciences, Beijing, 100093, People's Republic of China

Institute for Advanced Study, Tsinghua University, Beijing, 100084, People's RepubLIC OF CHINA

E-mail address: liu-mj07@mails.tsinghua.edu.cn

Department of Electrical Engineering and Computer Science, University of Wisconsin-Milwaukee, Milwaukee, Wisconsin 53201

E-mail address: gxu4uwm@uwm.edu 